\title{
Enhancing Presence - Immersive Sound Environments as Presence Generating Factor
}

\author{
Oliver Gingrich \\ CDE - Centre for Digital \\ Entertainment; Musion Systems \\ Bournemouth University, \\ Bath University, UK
}

\author{
Eugenia Emets \\ Musion Systems \\ London, UK
}

\author{
Alain Renaud \\ CDE - Centre for Digital Entertainment \\ Bournemouth University, \\ Bath University, UK
}

\begin{abstract}
This paper provides an overview of Kima - the Cymatic Musion Art project, a multi-modal networked installation, which suggests remote presence through the use of visualisation, remote sensing of sound and sound transformation. The installation is the outcome of research and work conducted by a team of artists and researchers with extensive experience in the field of telepresence. The paper firstly offers an introduction to Kima by outlining the artistic and technical objectives of the installation. Secondly, the paper offers a historical perspective of cymatics and telepresence and how both concepts relate to the installation. Thirdly, a technical overview of the installation is provided.
\end{abstract}

Cymatics. Telepresence. Holographic projection. Max MSP. Immersive sound. 5.1. Cladni plate. Motion control. Motion sensor. Musion. Remote sound. Installation art. Interactive art. Generative art.

\section{INTRODUCTION TO KIMA - ARTISTIC OBJECTIVES AND CONCEPTUAL FRAMEWORK}

This project intends to create a space offering an exploration of communication between two physically remote spaces / audiences using sound and visual feedback as means of communication.

Following Paul Watzlawick it is impossible not to communicate (Watzlawick 1967). Kima talks about the impossibility of non-communication. Information is exchanged constantly, but certain aspects are not visible or noticeable without instruments that render it perceivable. Telepresence enhances the sensation of somebody's presence or absence through two-way transmission of signals picked up in both spaces. Quantum physics discovered distant particles that synchronise across long distances as if they were communicating, as if they were "entangled". Kima builds on the conception of communication as such an "entanglement", but instead of simulating this phenomena, we look at communication as intersection of signals - through sonic and visual expression.

Two participants never see each other physically as there is no video feedback per se. They can only ever hear the voice that touches, provokes and drives their vocal, emotional and physical response.
They can feel the movement of the other in the movement of the sound through the speakers. Project "Kima" uses multiple speaker setup in order to map sound geometrically across these spaces, creating an immersive environment - a reference structure another participant in a similar environment. Sound of the two intersecting environments creates the communicative structure for movements of bodies. Intuitive interaction becomes conscious communication.

Immersive sound installation has a long tradition in contemporary art. Mapping sound across a space to navigate participants' experience has been explored for instance by John Wynne. Wynne explores both sound movement and a range of specific frequency mapping in his sound installations. Francisco Lopez creates immersive and partially sensory deprived (vision) environments for his performances with a multiple speaker setup.

Immersive sound installations have also been explored within the context of telepresence art: One of the early examples of art working with the idea of presence and telepresence is "Telematic Dreaming" by Paul Sermon (1992), a playful immersive experience with the video double presence of the other. "Lucifuge" a project by Yan Breuleux (Quebec) and Robin Meier (France) experiments with telepresence, robotic insects, and generative real-time sound. 
Dark space makes one in tune and aware of one's own movement, voice, intentions, as well as the other's presence. What is the other in the other space? There is no you and there is no me, but an endless echo that reverberates through both spaces - carried through the invisible network in the form of the coded information. The idea of 'Invisibility' has been explored in interactive art installations for instance by Jeppe Hein. Hein's "Invisible Labyrinth" explores the users' sense of limits and boundaries - virtual obstacles are revealed through "real" user's movements.

In "Kima" communication not only occurs between users, but also between users and the structure of their space. This invisible structure is represented by the physical dimensions of the space, as well as motion detection and the sound system that transmits frequencies and maps them to so users respond to a range of lower and higher vibrations in different ways.

More importantly, participants interact between these two physically distant spaces with one another. They are not able to see each other, but can navigate the space by moving through a soundscape, hence getting references on location of the other person from the sound itself. They can also refer to one another through each other's voices as mirroring echoes of their own. All they see and hear is not a direct signal, but an interference pattern of the communicative actions they perform themselves and the others interactions with themselves.

This communication happens in real time: Voices and the sounds of both spaces overlap and get modified so that both participants can only observe the result of their interaction as opposed to receiving immediate feedback from any of the two spaces.

In "Kima" this interference sound pattern is visualized in an abstract geometric form through a precise mathematical principle of sound aspects forming matter. Based on the idea of cymatics - the research of sound aspects influence on matter light projection forms a sculptural vision of a sonic sculpture of real time communication. The visual expression of this communication is based on its sonic pattern and the cymatic forms that emerge in real time from it.

A number of artists have been inspired and worked with the idea of cymatics. Among the most recent work that has been of influence to this project are two pieces by Paul Prudence and Ruth Jarman. Paul Prudence's project "BioAcousticPhenomena" (2010), is a live interactive visualization of bio acoustic phenomena. " $20 \mathrm{~Hz}$ " by Ruth Jarman and Joe Gerhardt is an artistic exploration of the visual patterns originating from data acquired in space. Alice Cunningham's " $90 \%$ Water" is a cymatic sound sculpture that visualizes cymatic phenomena. "Kima" distinguishes itself from these projects in that it is first and foremost a telepresence installation, and as such conceptualized as visualization of interference patterns, rather than mere representation of sound. In "Kima" cymatic interference patterns are projected into the space using a 3D holographic screen (Musion Eyeliner). Holographic projection functions as a mirror of this alternative state of communication, revealing highly abstracted aspects of a conversation between two people. One cannot see oneself in this reflection, but use it as a navigation interface to make a communicative connection to the other.

\section{CYMATICS AND TELEPRESENCE - HISTORIC AND THEORETIC BACKGROUND}

Musion's telepresence consists in a life-sized, perceived 3-dimensional telepresence setup that operates as a photorealistic real-time communication interface with a number of possible back-end codec transmission solutions. As a node to node network, it allows to stream life-sized, high definition signals of human-beings in perceived real-time from end to end. A Musion HD telepresence filming room serves as recording entity, a Musion Eyeliner screen as display unit. Heads-up displays depict reference signals for both - on-stage presenter and holographic presenter enabling users to communicate with unnoticeable latency as if standing on the same stage.

The term telepresence has experienced multiple transformation over the years - from a purely mechanical, robotic conception, it transformed into a terminology of virtual reality and ultimately became a function of everyday life. From Marvin Minsky's very technical, practise based, almost mechanical conception (Minsky 1980), to Thomas Sheridan's sensory-motoric and computer-centric approach (Sheridan 1992), to the prevailing perceptual definition as set out by the ISPR (ISPR 2000) - the theory of telepresence shows how varied conceptions of the term are. In practise, telepresence has multiple applications - from medicine, to engineering to boardroom conferences and not at least node to node communication in households worldwide. In 2011 Skype was purchased by Microsoft for USD \$ 8.7 billion representing only a fraction of the market volume of this emerging industry.

Musion telepresence was launched in 2008, after pilot setups with Cisco Systems' John Chamber who addressed an audience in Bangalore from San Jose in California in real time as hologram - an experiment that made it onto the cover of the Times 
of India. Ever since its commercial inauguration aired on German national TV as a direct live stream between London and Berlin-, Musion pepper's ghost based telepresence has experienced a number of optimisations, and technical enhancements. This research presents an effort to further these developments technically with the objective to generate an optimised sense of presence for user experience.

Examples of successful Musion life-sized telepresence experiments are numerous: A telepresence stream between London and Florida for Infocomm 2009 saw a streamed motion graphics overlay in sync with a real time presenter triggering motion graphics from multiple locations. Orange's HelloDemain (2011) event featured holographic presenters beamed into various on stage environments ranging from NYC skyline scenes to fictive jungle worlds. The future of telepresence will experience a number of such enhancements -from remote access augmented reality applications to motion capture data transmission to immersive sound environments. To test the effectiveness of these approaches on users, their usefulness in generating a sense of presence at a remote location, this research group conducted a test setup that implements an immersive sound environment within a non-stage environment.

The aim of this installation is not only to test perceptions of physical presence through immersive sound, but to understand how presence can be induced using surround sound as a mediator for remote location communication. Research on this subject has been minimal and inconclusive so far (compare: Lombard \& Ditton 1997). The purpose of this case study is to create an artistic experience that conveys "presence" through sound as well as to establish a research design to measure the influence of immersive sound on telepresence. Our research group has decided to approach this subject by using the framework of sound visualisations and cymatics as a point of departure.

The term cymatics, derived from Greek кũ $\mu \alpha$ "wave" was coined by Hans Jenny in 1967 and describes the study of visible sound and vibration, modal behaviour of liquids within contained environments under the exposure of certain harmonic frequencies. In the 1960s and 1970s Hans Jenny conducted a range of experiments using the Cladhni plate as laboratorial setup to discuss sound wave patterns in rigid bodies. Ernst Cladhni (1756-1827) is known as the father of acoustics, and credited as the first to experiment with frequencies and their vibration's effects on surfaces and volumes. His laboratory experiments were conducted using sand on confined surface areas. Hans Jenny optimized these physical studies and catalogued their regularities as organised patterns. His publications led to an indepth analysis of his studies by a series of scholars over the coming decades.

Research into cymatic wave patterns has been furthered by academics, musicians and artists. On an academic level these studies have been realised by György Kepes, Founder of the Center of Advanced Visual Studies at the MIT and many others. On a musical and music theoretical level, notable studies include $R$. Pellegrino, The Electronic Arts of Sound and Light (1983), Alvin Lucier and his composition "Queen of the South" or Lewis Skyes "Augmented tonoscope" which he presented at EVA 2011. Cymatic patterns have been analysed mathematically, for instance by Stewart and Colwell(1939), by Elmore and Head (1985) Paul Bourke, Research Associate Professor at the University of Western Australia and many others.

The analysis of Cladhni patterns describes movement, i.e. speed, and its derivative acceleration of two sine waves being generated within restricted confinements of a plate - be it rectangular or circular. Variables such as stiffness of the plate and its extension along with the frequency itself define the vibration. Modal points occur where the speed of vibrations equals zero. On a Cladhni plate these modal points are arranged along lines - depending on the frequency pitch.

The study of these phenomena helps to bridge the gap between the phonetic and the visual world - a single plate can act quite literally as a mediator between two different worlds - the phonetic and the aural.

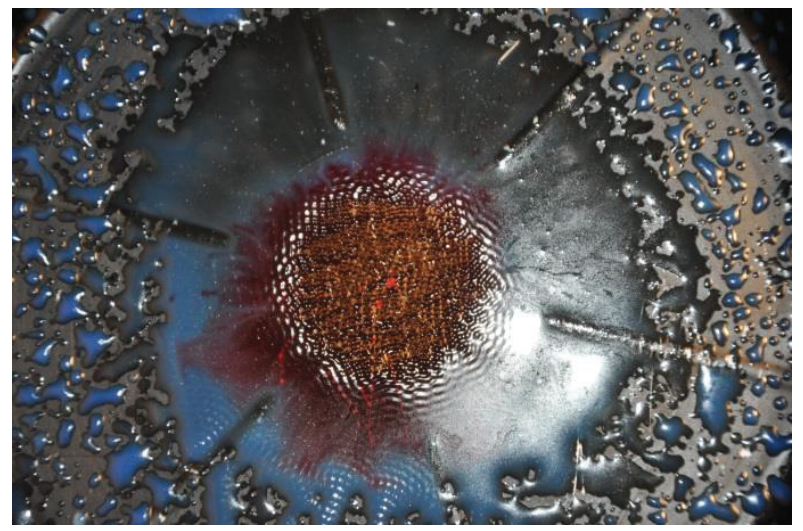

Figure 1: Cymatic installation by Eugenia Emets and Hello computer as part of Kima research 


\section{KIMA - RESEARCH OBJECTIVES}

Two main objectives guide the production process of Kima - our own experiments in audio-visual art: First and foremost, this study tries to establish a link between notions of presence and immersive sound environments. It has been suggested by researchers such as Lombard and Ditton (1997) that the quality of sound emphasises the conveyance of presence. Research by Kramer (1995) states that two key factors contribute to the emergence of presence - namely dimensionality and quality. However, the link between immersive sound environments and the evocation of presence is not an obvious one.

Although multi- dimensionality of sound is cited as relevant factor by Lombard and Ditton (Lombard \& Ditton 1997) and Short Williams and Christie (Christie 1976), relatively little hard evidence exists to support their thesis. Not only is research on the subject matter limited, it is also contradictory: Reeves, Detenber and Steuer (1993) found no difference in the perception of presence for surround and 2-dimensional sound. For the purpose of this study, we will compare two different setups: Setups using surround sound and setups using a 2-dimensional sound design so to be able to directly compare the two.

The second objective of this case study is to investigate the visual effect of sound patterns on wave structures using the java based programming language Processing (Reas, 2007) as an interface to Max/MSP(Cycling' 74, 2012) The starting point of our visual analysis are formulas derived directly from the mathematical formulas of Cladhni's law. Similar studies have been recently conducted by Graham Wakefield (Wakefield, 2009) at the University of California, Santa Barbara for Max/MSP. Our own research into the mathematical framework for our studies is helped by Ivan Isakov from University College London. Generative arts and its theoretical underpinnings build the artistic context for our research. Strongly associated with complex theory, generative arts focuses on the creation of semi-autonomous systems often using computer code.

Complexity theory points to an antipodal behaviour for the prevalence and number of rules to the complexity of visual outcome: The more constraining the rule set, the more ordered the visual outcome. Theories on cellular automata by Stephen Wolfram (Wolfram 2002) and others seem to support this school of thought. Generative art by definition points to a "closed" autonomous system in which a set of rules predefines the outcome of the artwork. In our research project, this constellation is interrupted by the viewers. Visual form is only ever being generated if two users interact and can be seen as the direct outcome of communication.

Generated forms are directly linked to the frequencies and wave patterns users generate through there very presence or by actively using microphones in their respective spaces. Motion control interfaces translate spatial information into sound patterns. The sonic space can be physically explored, altering generated aural patterns in realtime. The idea is not only to create a phonetic environment through surround sound, but to create a telematics experience of direct communication to induce a sense of presence that can be spatially discovered.

Kima creates two separate communication spaces - one of purely phonetic and one of pre-dominantly visual focus. Kima can be considered both - an immersive sound telepresence environment and an audio visual music instrument for two or many performers - it is at once academic experiment and performance space.
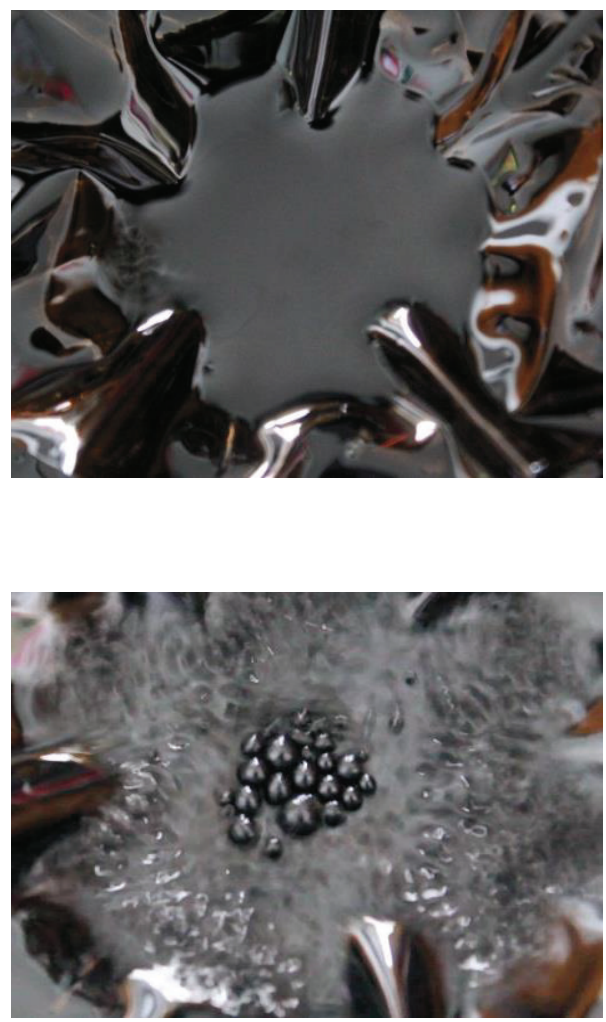

Figure 2: Before and After: Cymatic experiments using ferrofluids and the frequency range $432 \mathrm{khz}$ 


\section{KIMA - TECHNICAL OVERVIEW}

The Kima installation consists of three main aspects:

\subsection{An application to process audio information}

The Kima audio interface is the main hub of the system. The application was built with Max/Msp (Cycling' 74, 2012) and consists of a main control interface, a sample capture and processing interface and an OpenSoundControl (OSC) (Wright et. Al, 1997) broadcaster, which sends various messages over the network. Since the system is distributed over several sites, some of the audio signals are also sent via the network as raw audio data using the Jacktrip (Cáceres et. Al, 2009) software in conjunction with the JackAudioServer.

In the first space, the audio environment is captured through a combination of contact microphones, attached the structure of the space to capture the low rumbling frequencies and vibrations of the individuals in the space. In addition another condenser microphone captures the conversation and the noises emitted by the individuals in the space. Each sound source is processed in real time by the Max/Msp application. The sound sources are mixed automatically and played via an array of four speakers in the first space. The same mix is flattened as a two channel mix and sent to the other space via the network using Jacktrip. In the second space, one contact microphone and one condenser microphone capture the ambience and structure in the same fashion as in the first space. Each signal is sent individually to the Max/Msp application in the first space via Jacktrip and processed in the same fashion. The interferences created by comparing the various waveforms created in each space create the feeling of interferences as outlined earlier in the paper. The result is a natural sonic interaction between the two spaces. The overall sound generated is considered as an extension of the natural signals emitted by the individuals in each space and thus reinforce the feeling of interaction between the two spaces.

\subsection{A motion capture interface}

In the first space, using a Kinect motion sensor device, the approximate position of each participant is captured and mapped to the relative position (panning) of the processed sounds. This sound scape is processed in real time and transmitted to the secondary space where the other user can interact with it. Whereas one space has a predominantly phonetic interface, the other space features a visual representation of motion sensor data as well of their phonetic counterpart.

\subsection{A visual interface}

A set of OSC messages resulting from the audio processing taking place in the Max/Msp patch in the first space is sent out the network to animate a visual interface created using the Processing language.

The following parameters are rendered from the audio signal:

$$
\begin{array}{ll}
\text { - Amplitude } \\
\text { - Panning } \\
\text { - Frequency }
\end{array}
$$

This visual interface is based on the principles of cymatics - surface and volume waveform patterns generated by different frequencies in contained spaces. This visual representation follows formulas with an actual, physical counterpart and is based on algorithms that derive from water wave form patterns in a contained area as produced by a Cladhni plate for instance. Our visual interface is not primarily a phonetic representation, but a rule based autopoietic system influenced by, and influencing communication.

Seth Loyd and others have pointed to the fact that highly ordered generative art systems reduce visual entropy. Our telepresence interface is designed to ensure a mediated interaction that focuses on communication as immersive experience. To limit entropy, we chose a rule based approach for the Kima setup:

1) Kima is autonomous: The aim is to create a completely self-generative, autonomous and self-relying structure

2) Kima is cymatic: Both interfaces are based on principles of Cymatics (sound creates and alters visual form)

3) Kima is communicative: Interference patterns of communication are its visual and phonetic representation.

4) Kima is more than the sum of its parts: It consists of wave forms and particles that form together in entropy

5) Kima is audio-visual: The colour spectrum is related to frequencies, sound panning represents locations.

6) Kima is immersive: Physical space is mapped to a phonetic space and different effects are attributed to it

7) Kima is user focused: Visually and phonetically, centres are mapped to XYZcoordinates of users

8) Kima is generative: Audio loops entail repetition of the form

9) Kima is interactive: Once you stop to interact, the structure disappears

10) Kima is iconographic: Structure is being created around the centre 
11) KIma is representational: Amplitude of sound waves control particle emission

12) Kima is biologically inspired: Cladhni pattern principles are applied to a 3D space

Conceptually, the project is very much situated in the cross-over between interactive art and generative art: A rule based system acts an autonomous agent to facilitate communication between two people. The system is designed to display interference patterns. Humans act as music instruments, as their movements create phonetic soundscapes.

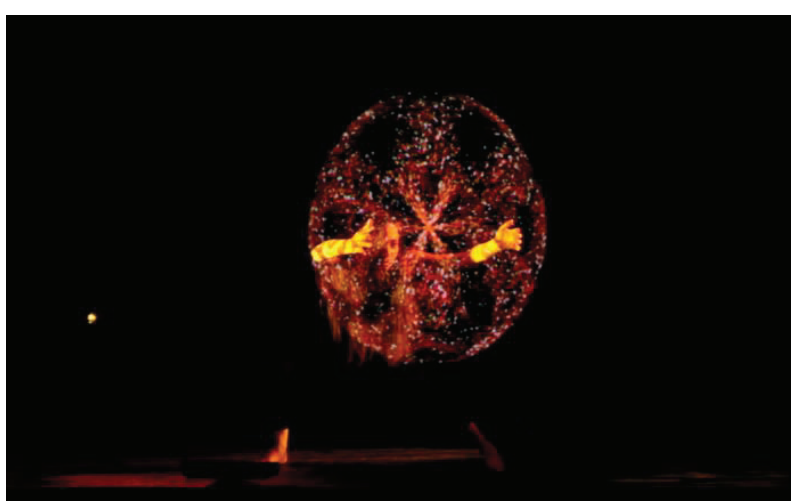

Figure 3: Screenshot from Kima performance at Kinetica Art Fair 2013

\section{CONCLUSIONS AND FUTURE WORK}

Kima is both a research project and an interactive art installation. This research group wants to specifically explore the influence of immersive sound environments on subjective and intersubjective telepresence experiences. Extensive research has been undertaken to study the concept of presence, its underlying factors, and contributing components. A qualitative analysis on the influence of sound environments stands at the end of this research project.

The authors understand the concept of presence as an immersive experience rather than just a face to face interaction. At the heart of this installation lies a dual approach: The exploration of non-verbal, and non-facial real-time communication - an interactive art installation, as well as a generative art work as an autonomous system based on rules drawn from the mathematical world of audio-visual phenomena.

In the tradition of generative arts, programs (along with machines, drawings etc.) play the role of autonomous systems, systems created using a set of rules to create intelligent design. In our case, these rules of wave patterns and their modal lines create a visual system that is not that autonomous per se: Quite the contrary, this system it is not only open for interaction, it needs interaction to come into being.

The tradition of interactive art engages users in an immersive experience that aims for un-mediated engagements. In the case of Kima mediation is the subject matter itself: Mediation is quite literally the subject and object of the art work, the condition sine qua non. Kima is conceptualised at the cross road between two adjacent art genres that are normally distinctively defined by their terminologies.

Apart from being a research project in telepresence, an interactive art installation and a generative art work, Kima is also a research project in wave length and audio-visual phenomena. In teaming up with experts from the field of physics and mathematics (Ivan Isakov from UCL) and sound artists (Patricia Afari), we want to ensure a truthfulness to physical conditions of audio-visual wave forms and their artefacts. In the tradition of Hans Jenny, Ernst Cladhni and cymatics, we are basing the code on real-time wave forms, exploring modal lines - the ever changing spaces where sound waves come to a stand-still. Modal lines form the geometric patterns of cymatic wave forms - they are not only interference patterns, but also the sum of points, in which waves do not transmit, or in our case where non-communication occurs for distinct moments in time.

Non-communication only ever occurs momentarily as non-communication is physically almost impossible. Kima makes these distinct moments in time graspable, and allows for a real-time telepresence experience that understands sound as an immersive environment, a world to explore, a world driven and generated by communication.

KIMA performance documentation at Kinetica 2013:

http://www.youtube.com/watch?v=YSQaGcWmW7 E

\section{REFERENCES}

Boden, M., Edmonds, E. (2010) What is Generative Art? Digital Creativity, 20:1-2,21-46 Routledge.

Bornstein, M. (1977) (Review): Symmetry in Science and Art by A. V. Shubnikov; V. A. Koptsik; G. D. Archard; In: Leonardo. Vol. 10, No. 4, p. 334 MIT Press.

Cáceres, J-P. and Chafe, C. (2009) JackTrip: Under the hood of an engine for network audio. In Proc. of International Computer Music Conference, Montreal.

Cunningham, Alice, http://www.cymatics.co.uk/alice-cunningham/ (retrieved 1 Mar 2013) 
Cycling '74. http://www.cycling74.com (retrieved 9 Nov 2012)

Dechevsky, L., Gundersen, J. (2009) On the Scientific Visualization of Complex-Valued Functions of One Complex Variable; Narvick University. In: Venkov, G., Kovacheva, R., Pasheva, V. (eds.): Applications of Mathematics in Engineering and Economics. American Institute of Physics.

Digitalarti Mag, "Digital Art and Innovation", http://www.digitalarti.com/blog/digitalarti mag/lucifu ge project telepresence and digital art (retrieved 3 Apr 2013)

Elmore, W.C. and Heald, M.A. (1985) Physics of Waves. Dover Publications. New York.

\section{Francisco Lopez}

http://www.sensitivebrigade.com/wynne.htm

(retrieved 1 Apr 2013)

JackAudio. http://jackaudio.org (retrieved 9 Nov 2012)

Jarman, R., and Gerhardt, J. "20Hz" project, http://www.semiconductorfilms.com/root $/ 20 \mathrm{~Hz} / 2 \mathrm{OH}$ z.htm (retrieved 10 Nov 2012)

Jenny, H. (1967) Cymatics: A Study of Wave Phenomena and Vibration Vol. 1.

Lombard, M., and Ditton, T. (1997) At the Heart of It All. The concept of Presence. Temple University.

Heeter, C. (1992) "Being There: The subjective experience of presence." Presence, 1(2), 262-271.

Hein, J. "Invisible Labyrinth", accessed at http://www.jeppehein.net/pages/project id.php?pat

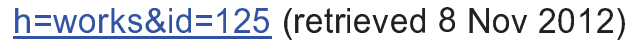

Lloyd, S. (2006) Programming the Universe: A Quantum Computer Scientist Takes on The Cosmos; New York.

Kramer, G. (1995). Sound and communication in virtual reality. In F. Biocca \& M. R. Levy (Eds.), Communication in the age of virtual reality, (pp. 259-276). Hillsdale, NJ: Lawrence Erlbaum.

Prudence, P. Visual Music Archive, "BioAcousticPhenomena", http://visualmusicarchive.org/works/w/bioacousticp henomena.html (retrieved 10 Nov 2012)

Pellegrino, R. (1983) The Electronic Arts of Sound and Light. Van Nostrand Reinhold New York.

Reas, C., Fry, B., and Maeda, J. (2007) Processing: A Programming Handbook for Visual Designers and Artists. The MIT Press.

Sermon, $\mathrm{P}$.

http://www.medienkunstnetz.de/refmovie.php?mov =226\&play=video (retrieved 3 Apr 2013)

Sheridan, T.B. (1992) Musings on telepresence and virtual presence. Presence, 1(1), 120-126.

Short, J. Williams, E., and Christie, B. (1976) The social psychology of telecommunications. London: Wiley.

Sykes, L. (2011) The Augmented Tonoscope. Manchester Institute for Research and Innovation in Art and Design (MIRIAD).

Steuer, J. (1995) Defining virtual reality: Dimensions determining telepresence. In Frank Biocca \& Mark R. Levy (eds.), Communication in the age of virtual reality (pp. 33-56). Hillsdale, NJ: Lawrence Erlbaum Associates.

Stuart, J.K., and Colwell, R.C. (1939) The Calculation of Chladni Patterns. In: J. Acoust. Soc. Am. Volume 11, Issue 1, pp. 147-151.

Watzlawick, P., Jackson, D, Beavin, and O'Hanlon, J. (1969) B\&B: Pragmatics of Human Communication. A study of interactional patterns, pathologies and paradoxes.

Wolfram, S (2002) A New Kind of Science. Wolfram Media.

Wright, M., and Freed, A. (1997) Open Sound Control: A New Protocol for Communicating with Sound Synthesizers, Proc. 1997 International Computer Music Conference, Thessaloniki.

Wynne, John

http://www.sensitivebrigade.com/wynne.htm (retrieved 2 Apr 2013) 